\title{
Numerical Study of Electromagnetic Aerodynamic Control of Hypersonic Vehicles
}

\author{
Nicholas J. Bisek* and Iain D. Boyd ${ }^{\dagger}$ \\ Department of Aerospace Engineering, University of Michigan, Ann Arbor, MI, 48109, USA \\ and \\ Jonathan Poggie \\ Air Force Research Laboratory, Wright-Patterson AFB, OH, 45433-7512, USA
}

\begin{abstract}
A computational study of several analytical test cases is carried out to verify the implementation of the low magnetic Reynolds number approximation into a hypersonic flow solver. An investigation in several electrical conductivity models, including solutions to Boltzmann's equation, is made to determine the appropriate method for approximating the flow's electrical conductivity, since the conductivity is an essential part of the magnetohydrodynamic approximations. These improvements will facilitate future computational studies of the effects of externally applied magnetic fields on plasma-assisted flow control devices and provide more physically accurate modeling of these techniques.
\end{abstract}

\section{Nomenclature}

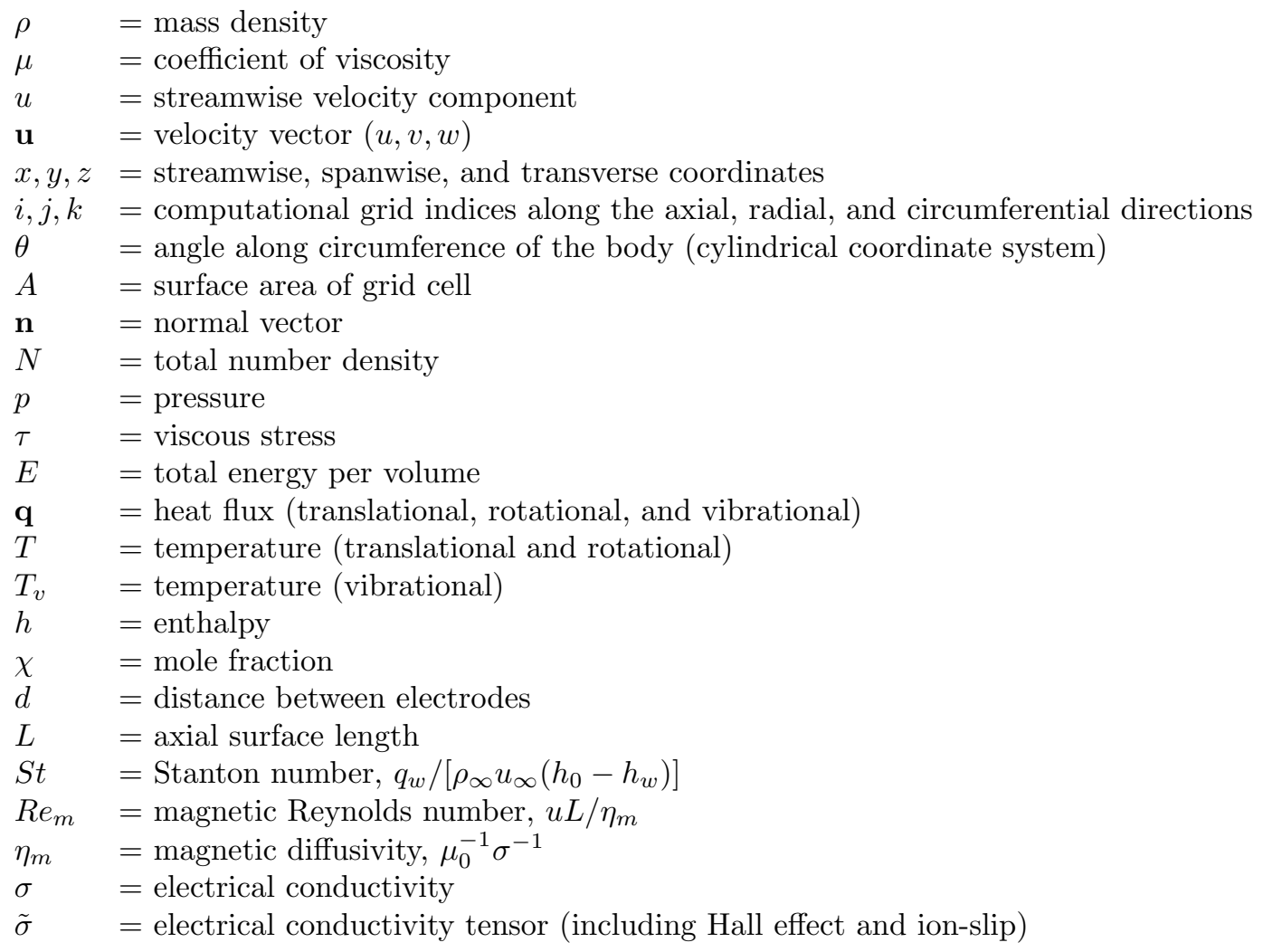

\footnotetext{
*Graduate Student, Student Member AIAA

${ }^{\dagger}$ Professor, Associate Fellow AIAA

${ }^{\ddagger}$ Senior Aerospace Engineer, AFRL/RBAC. Bldg. 146 Rm. 225, 2210 Eighth St. Associate Fellow AIAA
}

This material is declared a work of the U.S. Government and is not subject to copyright protection in the United States. 


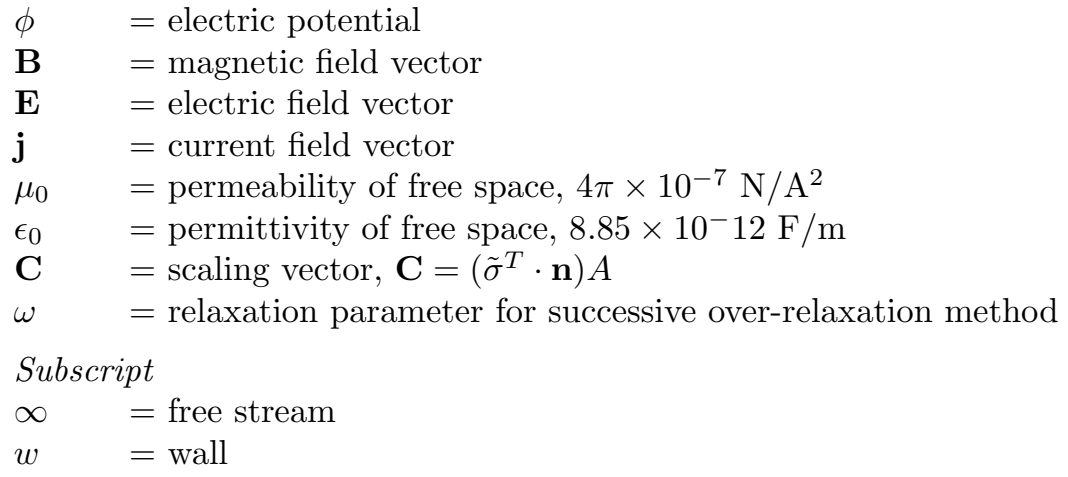

\section{Introduction}

Plasma-assisted hypersonic flow control has experienced a renewed interest over the past decade. ${ }^{1,2}$ This resurgence has been credited to many factors including the expanding requirements for sustained hypersonic flight and rapid access to space. These desires have produced several new challenges, particularly in airbreathing propulsion, where conventional means of flow control may be inadequate to meet the precise demands of scramjet and ramjet technologies. Plasma-based control systems are one of the options being explored to fill these needs. In addition to propulsion, these devices are also being applied to many other vehicle systems that could be enhanced by their abilities.

Recent experimental and computational research by Shang et al. ${ }^{3}$ has investigated ways of reducing drag on blunt nose bodies by means of plasma injection, while research by Kremeyer et al. ${ }^{4}$ and $\operatorname{Yan}^{5}$ focused on drag reduction and flow control using laser deposition (filamentation) ahead of conic and spherical geometries. Plasma actuators are being used to provide steering moments, ${ }^{6,7}$ changes in vehicle lift, ${ }^{8}$ control of flow separation,,${ }^{9,10}$ and in local heat load mitigation. ${ }^{11}$

A potential limitation of these devices is the large energy requirement necessary when they are employed to control large scale hypersonic flows by means of Joule heating through energy deposition. ${ }^{12}$ One possible way of improving the effectiveness and/or providing finer control is to utilize the ionized portion of the flow. The ionized flow can be subjected to electric and magnetic fields producing additional/improved flow control. In order to simulate these effects, computational fluid codes need to be modified to accurately account for the magnetohydrodynamic (MHD) effects. This is accomplished by solving some form of Maxwell's equations. Although it is possible to solve Maxwell's equations directly, ${ }^{13,14}$ most computational work in the area assumes the current continuity equation by means of the low magnetic Reynolds number approximation.

Barmin et al. demonstrated the need for 3D calculations when computing the MHD equations in order to prevent the introduction of unstable disturbances into the solution. ${ }^{15}$ MHD work by Gaitonde has included detailed high order numerical modeling ${ }^{16}$ and investigations into the Hall effect and ion-slip. ${ }^{17,18}$ Shang et al. ${ }^{19}$ investigated plasma actuators embedded at the entrance of a hypersonic inlet cowl, while Wan et al. studied MHD power generation. ${ }^{20}$ With DARPA's Falcon program entering its $3 \mathrm{rd} \mathrm{phase}^{21}$ and several other hypersonic test programs being conducted in other organizations and countries, ${ }^{22-25}$ the demand for continued development of computational tools in hypersonic flows continues to be strong.

The effort of this work is to implement the MHD effects into the 3D fluid solver used in previous work to quantify the required power expenditures for plasma-assisted devices in realistic hypersonic systems. ${ }^{12,26}$ In the following, we first provide a review of the flow code, LeMANS. The Lorentz force and Joule heating (MHD effects) are added to the conservation equations for a perfect gas solver. The low magnetic Reynolds number approximation is made to simplify Maxwell's equations in order to compute the electric and current fields. A solution to the MHD model is achieved using a finite volume, finite difference scheme. An examination of several electrical conductivity models is made, including results from a Boltzmann solver. Finally, solutions validating the MHD module are presented for an analytical case and for flow between two parallel electrode plates with a potential difference. 


\section{Method}

\section{A. Governing Equations}

Flow-field results are obtained using Computational Fluid Dynamics (CFD) to solve the Navier-Stokes equations. The CFD computations are executed using the Michigan Aerothermodynamic Navier-Stokes (LeMANS) code which was developed at the University of Michigan. ${ }^{27,28}$

LeMANS is a general 2D/axisymmetric/3D, parallel, unstructured finite-volume CFD code. The numerical fluxes between cells are discretized using a modified Steger-Warming Flux Vector Splitting (FVS) scheme, except near shock waves. In these regions the original Steger-Warming FVS scheme is used. LeMANS is able to employ a two-temperature model to account for thermal-nonequilibrium and a standard finite rate chemistry model for non-equilibrium chemistry. The two temperature model assumes that a single temperature $T$ accounts for the translational and rotational energy modes of all species while the vibrational energy mode is accounted for by a separate temperature $T_{v}$.

The usual conservation equations are solved:

$$
\begin{gathered}
\frac{\partial \rho}{\partial t}+\nabla \cdot(\rho \mathbf{u})=0 \\
\frac{\partial \rho \mathbf{u}}{\partial t}+\nabla \cdot\left(\rho \mathbf{u}^{2}+p \delta_{i j}-\tau\right)=\mathbf{j} \times \mathbf{B} \\
\frac{\partial E}{\partial t}+\nabla \cdot((E+p) \mathbf{u}-\tau \cdot \mathbf{u}-\mathbf{q})=\mathbf{j} \cdot \mathbf{E}
\end{gathered}
$$

LeMANS assumes the fluid is continuous and Newtonian. It also assumes Stokes' hypothesis when determining the viscous stresses.

$$
\tau_{i j}=\mu\left(\frac{\partial u_{j}}{\partial x_{i}}+\frac{\partial u_{i}}{\partial x_{j}}\right)-\frac{2}{3} \mu \nabla \cdot \mathbf{u} \delta_{i j}
$$

Previous work considered a phenomenological model of dissipative heating to account for a thermal actuator. ${ }^{26}$ This was represented as an additional source term on the right side of the energy equation (3), but is replaced with Joule heating $(\mathbf{j} \cdot \mathbf{E})$ in the equation's current form. The conservation of momentum equation is modified to include the Lorentz force $(\mathbf{j} \times \mathbf{B})$ on the right hand side of equation (2). These additions constitute the effects the electric and magnetic fields have on the flow. The vibrational energy equation is also modified with the inclusion of a Joule heating term $(\gamma \mathbf{j} \cdot \mathbf{j} / \sigma)$, where $\gamma$ is a factor that accounts for partitioning of electromagnetic energy deposition between different nonequilibrium modes. This equation is not included with the abovementioned governing equations because the remainder of the paper only employs a single temperature model and equilibrium chemistry.

The simulations are performed using second-order accurate spatial discretization and carry double precision arithmetic throughout. Thermal equilibrium and an eleven species, non-reacting air chemistry model $\left(\mathrm{N}_{2}, \mathrm{O}_{2}, \mathrm{NO}, \mathrm{N}, \mathrm{O}, \mathrm{N}_{2}^{+}, \mathrm{O}_{2}^{+}, \mathrm{NO}^{+}, \mathrm{N}^{+}, \mathrm{O}^{+}\right.$, and $e$ ) are used in the simulations, where $e$ is the electrons.

\section{B. Low Magnetic Reynolds Number Approximation}

The three additional variables appearing in the modified governing equations $(\mathbf{j}, \mathbf{B}, \mathbf{E})$ are determined by first noting that the magnetic Reynolds number, equation (5), is small for the cases of interest. Consequently, it can be shown that the induced magnetic field can be ignored. ${ }^{29}$ This means only external magnetic fields are present in the flow (and must be specified).

$$
R e_{m}=\frac{u L}{\eta_{m}}
$$

The current and electric field vectors are determined by starting with a generalized form of Ohm's law (adjusted for a flow moving relative to a magnetic field $\mathbf{B}$ ) which relates the two vectors as seen in equation (6). Following the outline in Ref. 30, the electrical conductivity tensor $\tilde{\sigma}$ is employed as a compact way of accounting for ion-slip and the Hall effect. The electrical conductivity tensor is further simplified by ignoring ion-slip and the Hall effect, which reduces the tensor to its scalar $\sigma$ multiplied by the identity matrix $(\tilde{\sigma}=\sigma I)$. 


$$
\mathbf{j}=\tilde{\sigma} \cdot(\mathbf{E}+\mathbf{u} \times \mathbf{B})
$$

Ampère - Maxwell's law, equation (7), is simplified by neglecting the current displacement term $\frac{\partial \mathbf{E}}{\partial t}$. This assumption is valid when its magnitude is compared to the conduction current $(\mathbf{j}=\sigma \mathbf{E})$ for typical conditions of air, see Ref. 31 for details. The reduced Ampère - Maxwell law is combined with charge conservation, equation (8), to yield the steady state form of the current continuity equation, equation (9).

$$
\begin{gathered}
\nabla \times \mathbf{B}=\mu_{0} \mathbf{j}+\mu_{0} \epsilon_{0} \frac{\partial \mathbf{E}}{\partial t} \\
\nabla \cdot \mathbf{j}=-\frac{\partial \rho}{\partial t} \\
\nabla \cdot \mathbf{j}=0
\end{gathered}
$$

Gauss's Law for magnetism $(\nabla \cdot \mathbf{B}=0)$ ensures the magnetic field is divergence free and absent of magnetic monopoles. This law is combined with the magnetic vector potential $(\mu \mathbf{B}=\nabla \times \mathbf{A})$ to prove that A (the vector potential) must exist.

Assuming the electric field vector is smooth and rapidly decaying, Helmholtz's theorem is used to decompose it into irrotational and divergence-free component vector fields. This is represented as a scalar potential $\phi$ and a vector potential $\mathbf{A} .(\mathbf{E}=-\nabla \phi+\nabla \times \mathbf{A})$ Using the low magnetic Reynolds number assumption, the right side of Faraday's law of induction, equation (10), must be zero for the external magnetic field specified in this work. Since $(\nabla \times \mathbf{E}=0)$, the electric field is irrotational (curl-free), so Helmholtz's decomposition of $\mathbf{E}$ is equation (11).

$$
\begin{gathered}
\nabla \times \mathbf{E}=-\frac{\partial \mathbf{B}}{\partial t} \\
\mathbf{E}=-\nabla \phi
\end{gathered}
$$

Combining equations $(6,9$, and 11) produces the Poisson type equation observed in equation (12).

$$
\nabla \cdot \tilde{\sigma} \cdot[-\nabla \phi+\mathbf{u} \times \mathbf{B}]=0
$$

To find the solution for $\phi$, and subsequently $\mathbf{E}$ and $\mathbf{j}$, a finite volume method is employed to be consistent with the flow solver. Rearranging the equation and integrating over an arbitrary volume yields

$$
\int_{V} \nabla \cdot[\tilde{\sigma} \cdot(\nabla \phi)] d V=\int_{V} \nabla \cdot[\tilde{\sigma} \cdot(\mathbf{u} \times \mathbf{B})] d V
$$

Applying the Divergence Theorem, equation (13) can be rearranged and written in a more compact form by introducing scaling vector $\mathbf{C}$ which is the sum of the face area multiplied with the dot product of the transpose of the electrical conductivity tensor and the outward facing normal as described in equation (14). The face area is $A$ and should not be confused with the vector potential. In $3 \mathrm{D}: \mathbf{C}=\left[\left(\sigma_{1,1} n_{x}+\sigma_{2,1} n_{y}+\right.\right.$ $\left.\left.\sigma_{3,1} n_{z}\right) A,\left(\sigma_{1,2} n_{x}+\sigma_{2,2} n_{y}+\sigma_{3,2} n_{z}\right) A,\left(\sigma_{1,3} n_{x}+\sigma_{2,3} n_{y}+\sigma_{3,3} n_{z}\right) A\right]$. Equating the surface integral to a sum over an arbitrary number of faces in a specific cell produces equation (15).

$$
\begin{gathered}
\mathbf{C}=\left(\tilde{\sigma}^{T} \cdot \mathbf{n}\right) A \\
\sum^{\text {faces }}(\nabla \cdot \phi) \cdot \mathbf{C}=\sum^{\text {faces }}(\mathbf{u} \times \mathbf{B}) \cdot \mathbf{C}
\end{gathered}
$$

During every iteration of LeMANS, a Poisson solver (MHD subroutine) is called to compute the electric and current field vectors by determining the electric potential $\phi$. With the velocity and magnetic field vectors provided by LeMANS, the right side of equation (15) is computed directly by approximating each face vector as a volumetric average of the adjoining cells. A finite difference approach is used to determine the electric potential flux $(\nabla \cdot \phi)$ through each face as seen in Figure 1. Following Refs. 32 and 33, a second order, nonuniform difference for the flux is written as 


$$
\frac{\partial \phi}{\partial x}=\frac{\phi_{\text {right }}+\left(\alpha^{2}-1\right) \phi_{\text {face }}-\alpha^{2} \phi_{\text {left }}}{\alpha(\alpha+1) \Delta x}
$$

where $\Delta x$ is the difference between the left cell center and the face center $\left(\Delta x=\left|x_{\text {face }}-x_{\text {left }}\right|\right)$ and $\alpha$ is the ratio of the right and left cell center differences $\left(\alpha=\left|\frac{x_{\text {face }}-x_{\text {right }}}{x_{\text {face }}-x_{\text {left }}}\right|\right)$. Equations (15 and 16) are applied to all cells using the Successive Over-Relaxation (SOR) iterative technique. The SOR technique is an iterative explicit solver that utilizes the direction of change to the solution to extrapolate an improved solution based on a relaxation constant $\omega$. The method converges more slowly than an implicit scheme, ${ }^{33}$ but is easier to implement and parallelize. For all computations presented here, $\omega=1.70$.

Mixed boundary conditions are generally employed at a solid surface. For electrodes that are good conductors, the potential is specified, either as a fixed value or determined by auxiliary equations representing an external circuit. For an insulated boundary, the normal component of current is set to zero: $\mathbf{j} \cdot \mathbf{n}=$ $\tilde{\sigma} \cdot(\mathbf{E}+\mathbf{u} \times \mathbf{B}) \cdot \mathbf{n}=0$. This can be a quite complicated boundary condition in the general case of tensor conductivity, but in the case of scalar conductivity and a no-slip wall, it reduces to $\mathbf{E} \cdot \mathbf{n}=0$.

The outward direction of symmetry plane must have a zero gradient electric field $(\mathbf{E} \cdot \mathbf{n}=0)$. By definition the ghost cell center lies on the outward normal vector, so the symmetry plane boundary condition is $\nabla \phi=0$. The proper boundary conditions in the far-field are less clear for aerodynamic MHD problems. For high accuracy, it may be necessary to solve the current continuity equation on a larger domain than the fluid conservation laws since the magnetic field can interact with the far-field. However, for external flows, the conductivity should drop to a negligible value far from the body such that it is assumed permissible to set the normal component of the electric field to zero. The normal component of the electric field is assumed to be very small at the inlet and is neglected $(\mathbf{E} \cdot \mathbf{n}=0)$. Finally, the outlet is assumed to be sufficiently downstream of the primary MHD interaction such that it is reasonable to set the normal component of the electric field to zero. The inlet and outlet boundary conditions are further simplified by their corresponding ghost cells $(\nabla \phi=0)$. Table 1 lists all domain boundaries and their respective conditions.

Table 1. Boundary conditions for MHD solver

\begin{tabular}{ccc}
\hline \hline Location & Type & Condition \\
\hline Inlet & Neumann & $\mathbf{E} \cdot \mathbf{n}=0$ \\
Far-field & Neumann & $\mathbf{E} \cdot \mathbf{n}=0$ \\
Symmetry & Neumann & $\mathbf{E} \cdot \mathbf{n}=0$ \\
Outlet & Neumann & $\mathbf{E} \cdot \mathbf{n}=0$ \\
Wall (electrode) & Dirichlet & $\phi=$ specified \\
Wall (insulating) & Neumann & $\mathbf{j} \cdot \mathbf{n}=0$ \\
\hline \hline
\end{tabular}

The Dirichlet conditions are directly substituted into the ghost cells joining the wall (electrode) boundary such that the wall face equals the specified value. The Neumann boundary conditions require the electric potential $\phi$ in the ghost cell to be determined iteratively in conjunction with the rest of the MHD solver. Since each ghost cell only has one face, they are updated to ensure $(\nabla \phi=0)$ for the face between the ghost cell and its adjoining real cell before the domain's interior cells are computed using equation (15).

\section{Electrical Conductivity}

\section{A. Equilibrium Air}

Over the years, equilibrium air properties have been the subject of several researchers. ${ }^{34-39}$ Research in this area continues to be further refined because it is necessary to accurately determine the correct thermal characteristics of a flow field, particularly in reacting flows like hypersonics. In addition to having the appropriate heat conduction, some electrical conductivity models are a function of the species mole fraction, another result of the flow field thermal characteristics. A range of input conditions to facilitate comparisons between various electrical conductivity models is generated utilizing computational research conducted by Godin and Trépanier ${ }^{40}$ to produce plots of the mole fractions of equilibrium air for a range of temperatures and pressures. 
Simulations are run for a temperature range of $10^{2}-10^{5} \mathrm{~K}$ with pressures ranging from $10^{-3}-1 \mathrm{~atm}$ for dry air (including $0.93 \%$ argon). Figure 2 shows the species mole fractions consistent with the eleven species computed in LeMANS $\left(\mathrm{N}_{2}, \mathrm{O}_{2}, \mathrm{NO}, \mathrm{N}, \mathrm{O}, \mathrm{N}_{2}^{+}, \mathrm{O}_{2}^{+}, \mathrm{NO}^{+}, \mathrm{N}^{+}, \mathrm{O}^{+}\right.$, and $e$ ). As seen in the figures, a pressure increase shifts the distributions to higher temperatures. Another noticeable result is the missing species at higher temperatures $(\mathrm{T}>15,000 \mathrm{~K})$. These missing species are primarily multi-ionized atomic nitrogen $\left(N^{++}, N^{3+}\right.$, and $\left.N^{4+}\right)$ as seen in Figure 3 and are usually neglected in hypersonic flow simulations because the post-shock flow field rarely achieves thermal equilibrium at these temperatures before exiting the domain.

\section{B. Conductivity Models}

As this research area has matured, several models have been developed to capture the behavior of the electrical conductivity in air which appears to be a function of both pressure and temperature as seen in Figure 4, reproduced from Sutton's Engineering Magnetohydrodynamics. ${ }^{31}$ Several empirical models only attempt to capture the conductivity once it starts to approach a constant (at high temperatures) because the conductivity is negligibly small at lower temperatures. Figure 5 is a photocopy from Cambel's text, Plasma Physics and Magnetofluid-Mechanics. It shows two such approximations for the electrical conductivity of argon at $\mathrm{p}=0.01 \mathrm{~atm} .{ }^{41}$ The Spitzer-Harm model, equation (17), was developed for fully ionized gases, whereas the model developed by Chapman and Cowling, equation (18), was developed for weakly ionized flows.

$$
\begin{gathered}
\sigma=\frac{1.56 \times 10^{-4} \times T^{1.5}}{\ln \left(1.23 \times 10^{4} \times T^{1.5} / n_{e}^{0.5}\right)} \Omega^{-1} \mathrm{~cm}^{-1} \\
\sigma=3.34 \times 10^{-12} \frac{\alpha}{Q T^{0.5}} \Omega^{-1} \mathrm{~cm}^{-1}
\end{gathered}
$$

where $\alpha$ is the degree of ionization and $Q$ is the collision cross section. Using research by Bush, ${ }^{42}$ Poggie and Gaitonde developed an electrical conductivity model that is only a function of temperature as seen in equation (19). ${ }^{43}$ This model has been adopted by others, including work by Otsu et al., ${ }^{44,45}$ which set $n=2$ (Poggie and Gaitonde set $n=4$ ). $\sigma_{0}$ is a reference conductivity and $T_{0}$ is the peak post-shock temperature. One set of reference values employed are $\sigma_{0}=731 \Omega^{-1} \cdot \mathrm{cm}^{-1}$ at $T_{0}=8000 \mathrm{~K}(\mathrm{p}=34 \mathrm{~Pa})$.

$$
\sigma=\sigma_{0}\left(\frac{T}{T_{0}}\right)^{n}
$$

Another weakly ionized model that is only a function of temperature was developed by Raizer. His method relates the conductivity to an exponential function as seen in equation (20), where $T$ is specified in Kelvin. This model is considered valid for air, nitrogen, and argon at $\mathrm{p}=1 \mathrm{~atm}$.

$$
\sigma=83 \times e^{-36000 / T} \Omega^{-1} \mathrm{~cm}^{-1}
$$

These semi-empirical models are compared against solutions to Boltzmann's equation using a Boltzmann solver developed by Weng and Kushner. ${ }^{46}$ The method of solving Boltzmann's equation is functionally equivalent to that proposed by Rockwood. ${ }^{47,48}$ Although the solver requires the translational temperature, pressure, and species mole fractions as input parameters, the solution of Boltzmann's equation only depends on E/N and each species mole fraction (E is the magnitude of the electric field and $N$ is the total number density). This means the input temperature and pressure are not adjusted to match the actual flow field. The solution is achieved using an extensive list of collision cross-section data taken from the compilations discussed in Refs. 49 and 50. The Boltzmann solver outputs the equilibrium transport coefficients for a range of $\mathrm{E} / N$ which are used to compute the electrical conductivity.

A disadvantage of computing solutions to Boltzmann's equation is that $\mathbf{E}$ is coupled to $\sigma$. This means the MHD solver depends on $\sigma$ to determine $\mathbf{E}$, see equation (12). Thus, the Boltzmann solver must be coupled to the MHD solver in order to compute $\sigma, \mathbf{E}$, and $\mathbf{j}$ simultaneously, which increases the computational cost of the simulation.

Once solutions of Boltzmann's equation are computed, $\sigma$ is determined using the resulting transport coefficients. Using the definition of conductivity for a dc current, equation (21) relates electrical conductivity to the electric charge $\left(q=1.6 \times 10^{-19} \mathrm{C}\right)$, the electron number density $\left(n_{e}\right)$, the electron mass $\left(9.11 \times 10^{-31}\right.$ 
$\mathrm{kg}$ ), and the electron momentum transfer collision frequency $\left(\nu_{m}\right)$. The electrical conductivity definition is combined with the definition of electron mobility, equation (22), to yield equation (23).

$$
\begin{aligned}
\sigma & =\frac{q^{2} n_{e}}{m_{e} \nu_{m}} \\
\mu & =\frac{q}{m_{e} \nu_{m}} \\
\sigma & =\mu q n_{e}
\end{aligned}
$$

The transport coefficients are given for values at standard conditions $(T=273 \mathrm{~K}, \mathrm{p}=1 \mathrm{~atm})$. The electrical conductivity is then computed for a given total number density $(N)$ by utilizing equation (24) which states the product of the electron mobility and total number density is a constant. This is substituted into equation (23) to yield equation (25) which relates the electrical conductivity to the electron mobility (a transport coefficient produced by solutions to Boltzmann's equation), and the degree of ionization.

$$
\begin{gathered}
\mu=\mu_{0}\left(\frac{N_{0}}{N}\right) \\
\sigma=\mu_{0} N_{0} q\left(\frac{n_{e}}{N}\right) \quad \Omega^{-1} c^{-1}
\end{gathered}
$$

\section{Conductivity Results}

To facilitate comparisons between the various electrical conductivity models, a list of species mole fractions for a range of pressures and temperatures is made using the plots in Figure 2. The table is listed in the Appendix (Table 2). For the Chapman-Cowling model, the degree of ionization $\alpha$ is the sum of the ionized species divided by the total number density $\left(\alpha=\Sigma n_{I} / N\right)$, and for simplicity, the collision cross-section is set equal to the vibrational cross-section for an ideal molecule $\left(Q=5 \times 10^{-17} \mathrm{~cm}^{2}\right)$.

The list of collision cross-section data available in the Boltzmann solver does not include $\mathrm{NO}^{+}$and must be neglected from the Boltzmann solutions. Fortunately, Table 2 and Figure 2 indicate the mole fraction of $\mathrm{NO}^{+}$is very low for all configurations being tested, so its absence is assumed inconsequential. For a low degree of ionization, the majority of the collisions are electron-neutral or electron-ion. As the degree of ionization increases, electron - electron collisions must be included, which dramatically increases the computational time of the solver.

Figures 6-8 show the electrical conductivity distributions of all the models versus temperature at $\mathrm{p}=$ $0.001,0.1$, and $1 \mathrm{~atm}$. The solid line shown for the Boltzmann solver is the conductivity for $\mathrm{E} / N=10$ Townsend ( $\mathrm{Td}$ ). (1 $\mathrm{Td}=10^{-17} \mathrm{~V} \mathrm{~cm}^{2}$ ) None of the semi-empirical models fully capture the behavior of the Boltzmann solver, although the Chapman-Cowling model appears to closely follow its behavior. This is because both the Chapman-Cowling model and Boltzmann solver directly depend on the degree of ionization.

While the semi-empiric models deviate from the electrical conductivity estimated by the Boltzmann solver, their reference parameters could be adjusted to better fit specific ranges of conditions. In addition, they are usually much easier to implement and computationally cheaper than a coupled MHD-Boltzmann approach. These advantages suggest the use of a semi-empirical model is a better choice for estimating the electrical conductivity used in the MHD solver as long as the model is valid for flow field conditions. However, large differences in the electrical conductivity (between the computed and real conductivity) will result in large changes in the current field and, subsequently, produce incorrect Lorentz force and Joule heating effects. Therefore, it is essential that care is taken when selecting reference parameters.

\section{MHD Results}

In previous work, LeMANS was validated for two different hypersonic cases. Simulations for hypersonic laminar air over elliptic blunt and sharp cones were compared to experimental work investigated by Nowlan et al..$^{51}$ and Kimmel et al. ${ }^{52,53}$ These experimental validation cases were selected because they are considered representative of a realistic hypersonic air-vehicle geometry and because of the limited amount of experimental data available. Figure 9 shows the experimental and computational Stanton number $(S t)$ distributions for the Mach 14 blunt elliptic cone investigated by Nowlan et al. ${ }^{51}$ Full details of the validation 
exercises and results are available in Refs. 12 and 26. Overall, LeMANS is capable of accurately computing three dimensional hypersonic laminar flows.

\section{A. MHD Validation Cases}

\section{Analytical case with Dirichlet Boundary Conditions}

Validation of the MHD module is accomplished by utilizing the analytical test case developed in the computational work by Gaitonde et al. ${ }^{30}$ This validation exercise has been utilized previously by Wan et $a l .,{ }^{20}$ and is selected for this work because it is simple, has magnetic and velocity field vectors, and an analytical solution. The governing equation for the test problem is

$$
\nabla^{2} \phi=x e^{z}
$$

Dirichlet boundary conditions are assigned to the outer cells for each side of the domain based on the analytical solution $\left(\phi=x e^{z}\right)$. To simplify the work, the electrical conductivity tensor is set to unity $(\tilde{\sigma}=1)$, although its exact value does not affect the solution. The magnetic and velocity field vectors are chosen as $\mathbf{B}=\left[0, \frac{1}{2} e^{z}, 0\right]$ and $\mathbf{u}=\left[u, 0, \frac{-1}{2} x^{2}\right]$ respectively.

Several meshes are employed including a uniform rectilinear, a nonuniform rectilinear (with cell clustering near $x=0, y=0, z=0$ ), and a rotated nonuniform rectilinear mesh, as seen in Figure 10. Each mesh has sides with length equal to one and the non-rotated meshes lie within the domain $0 \leq x \leq 1,0 \leq y \leq 1,0 \leq$ $z \leq 1$. The formal order of accuracy is estimated using the Richardson extrapolation function predefined in Tecplot $360 ®$. This method determines the formal order of accuracy by extrapolating the results from a sequence of meshes refined by a factor of two, i.e. the medium mesh has twice as many grid points on each side compared to the coarse mesh. For the cases examined, coarse $=10 \times 10 \times 10$, medium $=20 \times 20 \times 20$, and fine $=40 \times 40 \times 40$. The uniform mesh has a formal order of accuracy of 1.999 , which is expected since a 2nd order accurate finite difference scheme is employed. The nonlinear mesh produces a formal order of accuracy of 1.98 and the rotated mesh yields 1.80 due to the limitations of the finite difference approach employed.

Figure 11 shows the computed and analytical solutions for the rotated nonuniform mesh with part of the mesh removed to reveal the interior cells. The computed solutions are almost identical to the analytical solutions. Figure 12 plots the Least Squares Norm, for each of the meshes versus the number of iterations using the SOR method $(\omega=1.7)$. The uniform mesh requires more iterations to converge because $\omega$ is not optimized for its geometry and grid spacing.

\section{Flow Between Two Electrodes}

A second validation exercise is performed simulating flow between two parallel electrodes separated by a distance $d$, where $d$ is measured from the bottom electrode $(\mathrm{z}=0)$. Again, this validation exercise follows the computational work by Gaitonde et al. ${ }^{30}$ The electrodes have a specified potential in which the top electrode plate is equal to one and the bottom is set to zero $\left(\phi_{\text {top }}=1, \phi_{\text {bottom }}=0\right)$. Neumann boundary conditions are employed along the remaining sides of the domain so the normal component of the gradient is zero $\left(\frac{\partial \phi}{\partial n}=0\right)$. Figure 13 illustrates the domain with a rectilinear nonuniform mesh used in the simulation. For these grids, cell clustering is applied near both electrodes using a bi-exponential decay of cell size along the $z$ axis.

The simulations are computed assuming the velocity vector is zero $(\mathbf{u}=0)$ which significantly simplifies equation (12) to obtain a theoretical solution such that the current is constant $(\mathbf{j}=-\tilde{\sigma} \cdot \nabla \phi=$ constant). Two different electrical conductivity models are simulated. In the first case, $\sigma$ is constant $(\sigma=1)$, while the second case assumes the electrical conductivity diminishes as $d$ increases $\left(\sigma=1 / 2^{d}\right)$ as seen in Figure 14 .

For constant electrical conductivity $(\sigma=1)$, the theoretical solution reduces such that the gradient of the potential equals zero. The resulting electric potential is $\phi=z=d$ for the given boundary conditions. The theoretical solution for the second case is determined by recalling the Neumann boundary conditions applied to the four side walls, which indicate that the solution $\phi$ will not vary along the $x$ or $y$ axes. Since ion-slip and Hall effects are being neglected, the current equation reduces to

$$
j_{z}=\sigma \nabla_{z} \phi=2^{-d} \nabla_{z} \phi=\text { constant }=\mathrm{C}
$$


This equation can be further simplified by noting $d$ equals $z$ for the domain $\left(\frac{\partial \phi}{\partial z}=\mathrm{C} 2^{d}=\mathrm{C} 2^{z}\right)$. Integrating both sides over the domain and applying the electric potential from both plates yields

$$
\begin{gathered}
\int_{0}^{1} \frac{\partial \phi}{\partial z} d z=\int_{0}^{1} \mathrm{C} 2^{z} d z \\
\frac{\partial \phi}{\partial z}=\ln 22^{z}
\end{gathered}
$$

This is extended using each plate's electric potential to obtain the theoretical solution, which is written noting $d$ equals $z$ for the domain.

$$
\phi=2^{d}-1
$$

Figure 15 plots the computed and analytical solutions for the constant electrical conductivity problem on the nonuniform mesh with part of the mesh cut away to reveal the interior cells. Figure 16 plots the electric potential distributions along the $x=0.5, y=0.5$ ray for both electrical conductivity problems. The figures demonstrate that the MHD solver accurately computes the electric potential for the validation cases performed.

\section{Conclusions}

A low magnetic Reynolds number approximation was made to simplify Maxwell's equations in order to accurately account for electromagnetic effects for weakly ionized flows. A solution to the resulting MHD model was presented using a finite volume, finite difference scheme and coupled to LeMANS, an unstructured Navier-Stokes solver. Validation cases were successfully run for an analytical case and for flow between two parallel electrode plates with a potential difference.

In addition to the MHD solver, an examination of several electrical conductivity models was made. Although the semi-empiric models did not fully capture the behavior of the electrical conductivity distribution produced by the Boltzmann solver, they are valid for specific ranges of conditions and reference parameters. A more general approach would be to couple the Boltzmann solver to the MHD module. While this increases the computational cost of the simulation, the generality of the approach makes it attractive.

\section{Future Work}

In further studies, we will focus on the parallelization of the MHD solver and the MHD effects for thermal nonequilibrium flows. This includes modifications to the mass diffusion terms within the conservation equations (finite chemistry). These improvements will be used to investigate whether additional enhancements to the flow field can be achieved by introducing a magnetic field to the flow over a realistic hypersonic vehicle which is using an arc discharge between a cathode and anode on the vehicle's surface.

\section{Acknowledgments}

The authors are indebted to the Michigan/AFRL/Boeing Collaborative Center in Aeronautical Sciences which provides funding to the first author. The first author would like to thank Alexandre Martin for numerous discussions on equilibrium air properties and Mark Kushner for his valuable discussions on electrical conductivity and the use of his Boltzmann solver.

\section{References}

\footnotetext{
${ }^{1}$ Shang, J. S., Surzhikov, S. T., Kimmel, R., Gaitonde, D., Menart, J., and Hayes, J., "Mechanisms of Plasma Actuators for Hypersonic Flow Control," Progress in Aerospace Sciences, Vol. 41, No. 8, November 2005, pp. 642-668.

${ }^{2}$ Fomin, V. M., Tretyakov, P. K., and Taran, J.-P., "Flow Control using Various Plasma and Aerodynamic Approaches," Aerospace Science and Technology, Vol. 8, No. 5, July 2004, pp. 411-421.

${ }^{3}$ Shang, J. S., Hayes, J., and Menart, J., "Hypersonic Flow over a Blunt Body with Plasma Injection," Journal of Spacecraft and Rockets, Vol. 39, No. 3, May-June 2002, pp. 367-375.

${ }^{4}$ Kremeyer, K., Sebastian, K., and Shu, C.-W., "Computational Study of Shock Mitigation and Drag Reduction by Pulsed Energy Lines," AIAA Journal, Vol. 44, No. 8, August 2006, pp. 1720-1731.

${ }^{5}$ Yan, H. and Gaitonde, D., "Control of Edney IV Interaction by Energy Pulse," 44th AIAA Aerospace Sciences Meeting and Exhibit, AIAA Paper 2006-562, 2006.
} 
${ }^{6}$ Girgis, I. G., Shneider, M. N., Macheret, S. O., Brown, G. L., and Miles, R. B., "Creation of Steering Moments in Supersonic Flow by Off-Axis Plasma Heat Addition," 40th AIAA Aerospace Sciences Meeting and Exhibit, 2002, AIAA Paper 2002-129

${ }^{7}$ Gnemmi, P., Charon, R., Dupéroux, J.-P., and George, A., "Feasibility Study for Steering a Supersonic Projectile by a Plasma Actuator," AIAA Journal, Vol. 46, No. 6, June 2008, pp. 1308-1317.

${ }^{8}$ Menart, J., Stanfield, S., Shang, J., Kimmel, R., and Hayes, J., "Study of Plasma Electrode Arrangements for Optimum Lift in a Mach 5 Flow," 44th AIAA Aerospace Sciences Meeting and Exhibit, 2006, AIAA Paper 2006-1172.

${ }^{9}$ Updike, G. A., Shang, J. S., and Gaitonde, D. V., "Hypersonic Separated Flow Control Using Magneto-Aerodynamic Interaction," 43th AIAA Aerospace Sciences Meeting and Exhibit, AIAA Paper 2005-164, 2005.

${ }^{10}$ Kimmel, R. L., Hayes, J. R., Crafton., J. W., Fonov, S. D., Menart, J., and Shang, J., "Surface Discharge for High-Speed Boundary Layer Control," 44th AIAA Aerospace Sciences Meeting and Exhibit, AIAA Paper 2006-710, 2006.

${ }^{11}$ Miles, R. B., Macheret, S. O., Shneider, M. N., Steeves, C., Murray, R. C., Smith, T., and Zaidi, S. H., "PlasmaEnhanced Hypersonic Performance Enabled by MHD Power Extraction," 43th AIAA Aerospace Sciences Meeting and Exhibit, AIAA Paper 2005-561, 2005.

${ }^{12}$ Bisek, N. J., Boyd, I. D., and Poggie, J., "Numerical Study of Plasma-Assisted Aerodynamic Control for Hypersonic Vehicles," 39th AIAA Plasmadynamics and Lasers Conference, AIAA Paper 2008-4226, 2008.

${ }^{13}$ MacCormack, R., "Numerical Simulation of Aerodynamic Flow Including Induced Magnetic and Electric Fields," 39th Plasmadynamics and Lasers Conference, AIAA Paper 2008-4010, 2008.

${ }^{14}$ D'Ambrosio, D. and Giordano, D., "Two-Dimensional Numerical Methods in Electromagnetic Hypersonics Including Fully Coupled Maxwell Equations," 39th Plasmadynamics and Lasers Conference, AIAA Paper 2008-4013, 2008.

${ }^{15}$ Barmin, A. A., Kulikovskiy, A. G., and Pogorelov, N. V., "Shock-Capturing Approach and Nonevolutionary Solutions in Magnetohydrodynamics," Journal of Computational Physics, Vol. 126, No. 1, June 1996, pp. 77-90.

${ }^{16}$ Gaitonde, D. V., "A High-Order Implicit Procedure for the 3-D Electric Field in Complex Magnetogasdynamic Simulations," Computers and Fluids, Vol. 33, No. 3, March 2004, pp. 345-374.

${ }^{17}$ Gaitonde, D. V. and Poggie, J., "An implicit Technique for 3-D Turbulent MGD with the Generalized Ohm's Law," 32th AIA A Plasmadynamics and Lasers Conference, AIAA Paper 2001-2736, 2001.

${ }^{18}$ Gaitonde, D. V., "Effect of Hall Currents on Simulated Three-Dimensional Scramjet with Magnetohydrodynaic Bypass," Journal of Propulsion and Power, Vol. 22, No. 3, May-June 2006, pp. 700-703.

${ }^{19}$ Shang, J. S., Huang, H. Y., Surzhikov, S. T., and Gaitonde, D. V., "Hypersonic Flow Control Utilizing ElectronmaggeticAerodynamic Interaction," 15th AIAA International Space Planes and Hypersonic Systems and Technologies Conference, AIAA Paper 2008-2606, 2008.

${ }^{20}$ Wan, T., Suzuki, R., Candler, G., Macheret, S., and Schneider, M., "Three Dimensional Simulation of Electric Field and MHD Power Generation During Re-Entry," 36th AIAA Plasmadynamics and Lasers Conference, AIAA Paper 2005-5045, 2005.

${ }^{21}$ Walker, S., Tang, M. T., Morris, S., and Mamplata, C., "Falcon HTV-3X - A Reusable Hypersonic Test Bed," $15 t h$ AIA A International Space Planes and Hypersonic Systems and Technologies Conference, AIAA Paper 2008-2544, 2008.

${ }^{22}$ Fujii, K. and Ishimoto, S., "Research Activities to Realize Advanded Space Transportation System," $15 t h$ AIAA International Space Planes and Hypersonic Systems and Technologies Conference, AIAA Paper 2005-2575, 2008.

${ }^{23}$ Mutzman, R., Murphy, J., and Hank, J., "X-51A Scramjet Engine Flight Demonstration Program," 15th AIAA International Space Planes and Hypersonic Systems and Technologies Conference, AIAA Paper 2005-2540, 2008.

${ }^{24}$ Falempin, F. and Serre, L., "French LEA Flight Test Program: Status in 2008," 15th AIAA International Space Planes and Hypersonic Systems and Technologies Conference, AIAA Paper 2005-2541, 2008.

${ }^{25}$ Weihs, H., Longo, J., and Turner, J., "Sharp Edge Flight Experience SHEFEX II: A Mission Overview and Status," 15th AIAA International Space Planes and Hypersonic Systems and Technologies Conference, AIAA Paper 2005-2542, 2008.

${ }^{26}$ Bisek, N. J., Boyd, I. D., and Poggie, J., "Numerical Study of Energy Deposition Requirements for Aerodynamic Control of Hypersonic Vehicles," 46nd AIAA Aerospace Sciences Meeting and Exhibit, AIAA Paper 2008-1109, 2008.

${ }^{27}$ Scalabrin, L. C. and Boyd, I. D., "Development of an Unstructured Navier-Stokes Solver For Hypersonic Nonequilibrium Aerothermodynamics," 38th AIAA Thermophysics Conference, AIAA Paper 2005-5203, 2005.

${ }^{28}$ Scalabrin, L. C. and Boyd, I. D., "Numerical Simulation of Weakly Ionized Hypersonic Flow for Reentry Configurations," 9th AIAA/ASME Joint Thermophysics and Heat Transfer Conference, AIAA Paper 2006-3773, 2006.

${ }^{29}$ Shercliff, J., A Textbook of Magnetohydrodynamics, Pergamon Press, 1965.

${ }^{30}$ Gaitonde, D. V. and Poggie, J., "Elements of a Numerical Procedure for 3-D MGD Flow Control Analysis," 40th AIAA Aerospace Sciences Metting and Exhibit, AIAA Paper 2002-198, 2002.

${ }^{31}$ Sutton, G. W. and Sherman, A., Engineering Magnetohydrodynamics, McGraw-Hill, 1965.

${ }^{32}$ Hoffman, K. A. and Chiang, S. T., Computational Fluid Dynamics - Vol. 1, 4th ed., Engineering Education System, 2000.

${ }^{33}$ Tannehill, J. C., Anderson, D. A., and Pletcher, R. H., Computational Fluid Mechanics and Heat Transfer, 2nd ed., Hemisphere Publishing Corporation, 1997.

${ }^{34}$ Hansen, C., "Approximations for the Thermodynamic and Transport Properties of High-Temperature Air," Tech. Rep. TR-R-50, NASA, 1959.

${ }^{35}$ Srinivasan, S., Tannehill, J., and Weilmuenster, K., "Simplified Curve Fits for the Thermodynamic Properties of Equilibrium Air," Tech. Rep. RP-1181, NASA, 1987.

${ }^{36}$ Gupta, R. N., Lee, K.-P., Thompson, R. A., and Yos, J. M., "Calculation and Curve Fits of Thermodynamic and Transport Properties for Equilibrium Air to 30,000 K," Tech. Rep. RP-1260, NASA, 1991.

${ }^{37}$ Sokolova, I. A., "Transport Properties of Weakly Ionized Air," 32nd AIAA Plasmadynamics and Lasers Conference and 4 th Weakly Ionized Gases Workshop, AIAA Paper 2001-3083, 2001. 
${ }^{38}$ D'Angola, A., Colonna, G., Gorse, C., and Capitelli, M., "Thermodynamic and Transport Properties in Equilibrium Air Plasmas in a Wide Pressure and Temperature Range," The European Physical Journal D, Vol. 46, No. 1, January 2008, pp. 129-150.

${ }^{39}$ Henderson, S. J. and Menart, J. A., "Equilibrium Properties of High-Temperature Air for a Number of Pressures," Journal of Thermophysics and Heat Transfer, Vol. 22, No. 4, October 2008, pp. 718-726.

${ }^{40}$ Godin, D. and Trépanier, J. Y., "A Robust and Efficient Method for the Computation of Equilbrium Composition in Gaseous Mixtures," Plasma Chemistry and Plasma Processing, Vol. 24, No. 3, September 2004, pp. $447-473$.

${ }^{41}$ Cambel, A. B., Plasma Physics and Magnetofluid-Mechanics, McGraw-Hill, 1963, p. 171.

${ }^{42}$ Bush, W. B., "The Stagnation-Point Boundary Layer in the Presence of an Applied Magnetic Field," Journal of Aerospace Sciences, Vol. 28, No. 8, August 1961, pp. 610-611, 630.

${ }^{43}$ Poggie, J. and Gaitonde, D. V., "Computational Studies of Magnetic Control in Hypersonic Flow," 39th Aerospace Sciences Meeting and Exhibit, AIAA Paper 2001-0196, 2001.

${ }^{44}$ Otsu, H., Abe, T., and Konigorski, D., "Influence of the Hall Effect on the Electrodynamic Heat Shield System for Reentry Vehicles," 36th AIAA Plasmadynamics and Lasers Conference, AIAA Paper 2005-5049, 2005.

${ }^{45}$ Otsu, H., Matsuda, A., Abe, T., and Konigorski, D., "Numerical Validation of the Magnetic Flow Control for Reentry Vehicles," 37th AIAA Plasmadynamics and Lasers Conference, AIAA Paper 2006-3236, 2006.

${ }^{46}$ Weng, Y. and Kushner, M. J., "Method for Including Electron-Electron Collisions in Monte Carlo Simulations of Electron Swarms in Partially Ionized Gases," Physical Review A, Vol. 42, No. 10, November 1990, pp. 6192-6200.

${ }^{47}$ Rockwood, S. D., "Elastic and Inelastic Cross Sections for Electron-Hg Scattering from Hg Transport Data," Physical Review A, Vol. 8, No. 5, November 1973, pp. 2348-2358.

${ }^{48}$ Rockwood, S. D., "Effect of Electron-Electron and Electron-Ion Collisions in $\mathrm{Hg}, \mathrm{CO}_{2} / \mathrm{N}_{2} / \mathrm{He}$, and $\mathrm{CO} / \mathrm{N}_{2}$ discharges," Journal of Applied Physics, Vol. 45, No. 12, December 1974, pp. 5229-5234.

${ }^{49}$ Dorai, R. and Kushner, M. J., "A Model for Plasma Modification of Polypropylene using Atmospheric Pressure Discharges," Journal of Physics D: Applied Physics, Vol. 36, No. 6, March 2003, pp. 666-685.

${ }^{50}$ Stafford, D. S. and Kushner, M. J., "O $\mathrm{O}_{2}\left({ }^{1} \Delta\right)$ Production in $\mathrm{He} / \mathrm{O}_{2}$ mixtures in Flowing Low Pressure Plasmas," Journal of Applied Physics, Vol. 96, No. 5, September 2004, pp. 2451-2465.

${ }^{51}$ Nowlan, D., Burke, A., and Bird, K., "Pressure and Heat Transfer Distribution on ASD Elliptic Cone (W3) and ASD Sortie (W4) in the CAL 48-Inch Hypersonic Shock Tunnel," Tech. Rep. AM-1800-Y-2, Cornell Aeronautical Laboratory, Inc., Buffalo, NY, December 1963, ASC 942649.

${ }^{52}$ Kimmel, R. L., Poggie, J., and Schwoerke, S. N., "Laminar-Turbulent Transition in a Mach 8 Elliptic Cone Flow," AIAA Journal, Vol. 37, No. 9, September 1999, pp. 1080-1087.

${ }^{53}$ Kimmel, R., Klein, M., and Schwoerke, S., "Three-Dimensional Hypersonic Laminar Boundary-Layer Computations for Transition Experiment Design," AIAA Journal, Vol. 34, No. 4, July-August 1997, pp. 409-415. 


\section{Appendix}

Table 2. Temperature, pressure, and mole fractions for air computed using the computational code cited in Ref. 40. $\left(\chi=N_{2} / O_{2} / N O / N / O / N_{2}^{+} / O_{2}^{+} / N^{+} / N^{+} / O^{+} / e\right)$

\begin{tabular}{lcc}
\hline \hline Pressure & Temperature & Mole Fractions $[\chi]$ \\
\hline $0.001 \mathrm{~atm}$ & $2000 \mathrm{~K}$ & $0.80 / 0.18 / 0.01 / 0.00 / 0.01 / 0.00 / 0.00 / 0.00 / 0.00 / 0.00 / 0.00$ \\
$0.001 \mathrm{~atm}$ & $4000 \mathrm{~K}$ & $0.64 / 0.00 / 0.00 / 0.04 / 0.31 / 0.00 / 0.00 / 0.00 / 0.00 / 0.00 / 0.00$ \\
$0.001 \mathrm{~atm}$ & $6000 \mathrm{~K}$ & $0.01 / 0.00 / 0.00 / 0.79 / 0.19 / 0.00 / 0.00 / 0.00 / 0.00 / 0.00 / 0.00$ \\
$0.001 \mathrm{~atm}$ & $8000 \mathrm{~K}$ & $0.00 / 0.00 / 0.00 / 0.70 / 0.17 / 0.00 / 0.00 / 0.00 / 0.06 / 0.01 / 0.07$ \\
$0.001 \mathrm{~atm}$ & $10000 \mathrm{~K}$ & $0.00 / 0.00 / 0.00 / 0.19 / 0.06 / 0.00 / 0.00 / 0.00 / 0.32 / 0.06 / 0.38$ \\
$0.001 \mathrm{~atm}$ & $12000 \mathrm{~K}$ & $0.00 / 0.00 / 0.00 / 0.01 / 0.01 / 0.00 / 0.00 / 0.00 / 0.40 / 0.09 / 0.49$ \\
$0.100 \mathrm{~atm}$ & $2000 \mathrm{~K}$ & $0.81 / 0.19 / 0.01 / 0.00 / 0.00 / 0.00 / 0.00 / 0.00 / 0.00 / 0.00 / 0.00$ \\
$0.100 \mathrm{~atm}$ & $4000 \mathrm{~K}$ & $0.68 / 0.00 / 0.02 / 0.00 / 0.30 / 0.00 / 0.00 / 0.00 / 0.00 / 0.00 / 0.00$ \\
$0.100 \mathrm{~atm}$ & $6000 \mathrm{~K}$ & $0.32 / 0.00 / 0.00 / 0.42 / 0.25 / 0.00 / 0.00 / 0.00 / 0.00 / 0.00 / 0.00$ \\
$0.100 \mathrm{~atm}$ & $8000 \mathrm{~K}$ & $0.01 / 0.00 / 0.00 / 0.79 / 0.19 / 0.00 / 0.00 / 0.00 / 0.01 / 0.00 / 0.01$ \\
$0.100 \mathrm{~atm}$ & $10000 \mathrm{~K}$ & $0.00 / 0.00 / 0.00 / 0.69 / 0.17 / 0.00 / 0.00 / 0.00 / 0.06 / 0.01 / 0.07$ \\
$0.100 \mathrm{~atm}$ & $12000 \mathrm{~K}$ & $0.00 / 0.00 / 0.00 / 0.36 / 0.10 / 0.00 / 0.00 / 0.00 / 0.23 / 0.03 / 0.27$ \\
$1.000 \mathrm{~atm}$ & $2000 \mathrm{~K}$ & $0.81 / 0.19 / 0.01 / 0.00 / 0.00 / 0.00 / 0.00 / 0.00 / 0.00 / 0.00 / 0.00$ \\
$1.000 \mathrm{~atm}$ & $4000 \mathrm{~K}$ & $0.69 / 0.03 / 0.04 / 0.00 / 0.24 / 0.00 / 0.00 / 0.00 / 0.00 / 0.00 / 0.00$ \\
$1.000 \mathrm{~atm}$ & $6000 \mathrm{~K}$ & $0.53 / 0.00 / 0.01 / 0.17 / 0.29 / 0.00 / 0.00 / 0.00 / 0.00 / 0.00 / 0.00$ \\
$1.000 \mathrm{~atm}$ & $8000 \mathrm{~K}$ & $0.06 / 0.00 / 0.00 / 0.73 / 0.20 / 0.00 / 0.00 / 0.00 / 0.00 / 0.00 / 0.00$ \\
$1.000 \mathrm{~atm}$ & $10000 \mathrm{~K}$ & $0.00 / 0.00 / 0.00 / 0.76 / 0.18 / 0.00 / 0.00 / 0.00 / 0.02 / 0.00 / 0.02$ \\
$1.000 \mathrm{~atm}$ & $12000 \mathrm{~K}$ & $0.00 / 0.00 / 0.00 / 0.62 / 0.16 / 0.00 / 0.00 / 0.00 / 0.10 / 0.01 / 0.11$ \\
\hline \hline
\end{tabular}




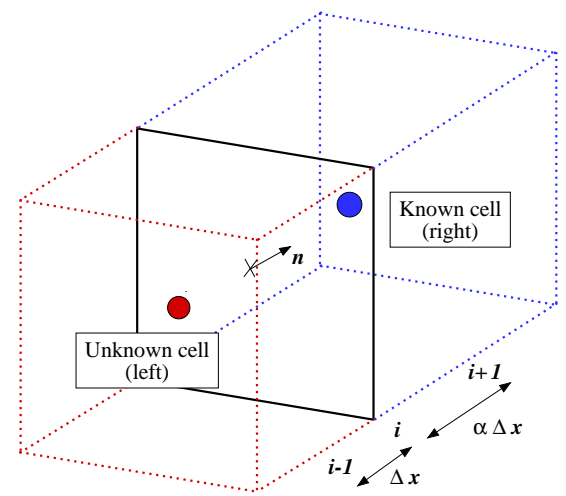

Figure 1. An illustration of the nonuniform finite difference methodology used to find the flux through face $i$ from $i-1$ to $i+1$. ( $\phi$ is known at $i+1$ [blue] and unknown at $i-1$ [red])

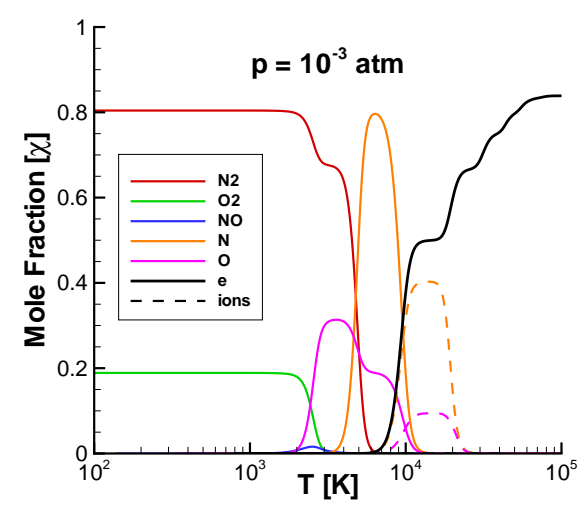

(a) $\mathrm{p}=0.001 \mathrm{~atm}$

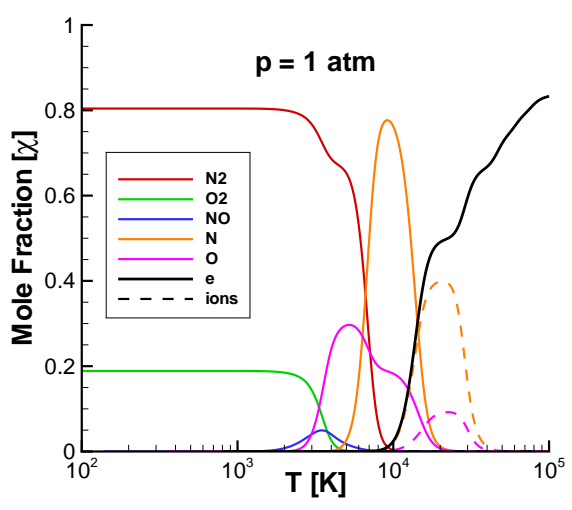

(b) $\mathrm{p}=1.0 \mathrm{~atm}$

Figure 2. Mole fractions of equilibrium air versus temperature for various pressures. (11 species)

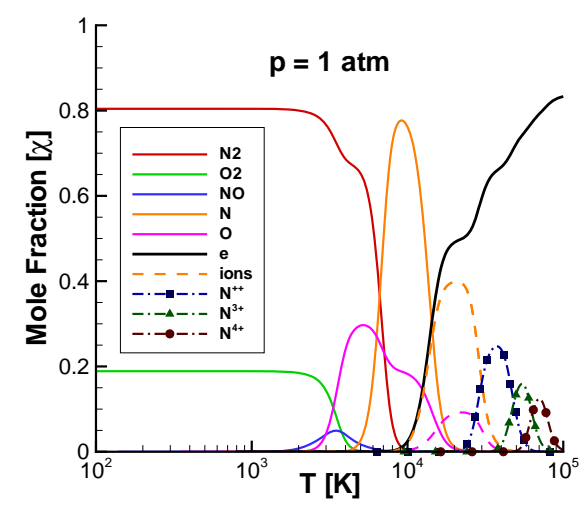

Figure 3. Mole fractions of equilibrium air versus temperature for $p=1$ atm. (14 species) 


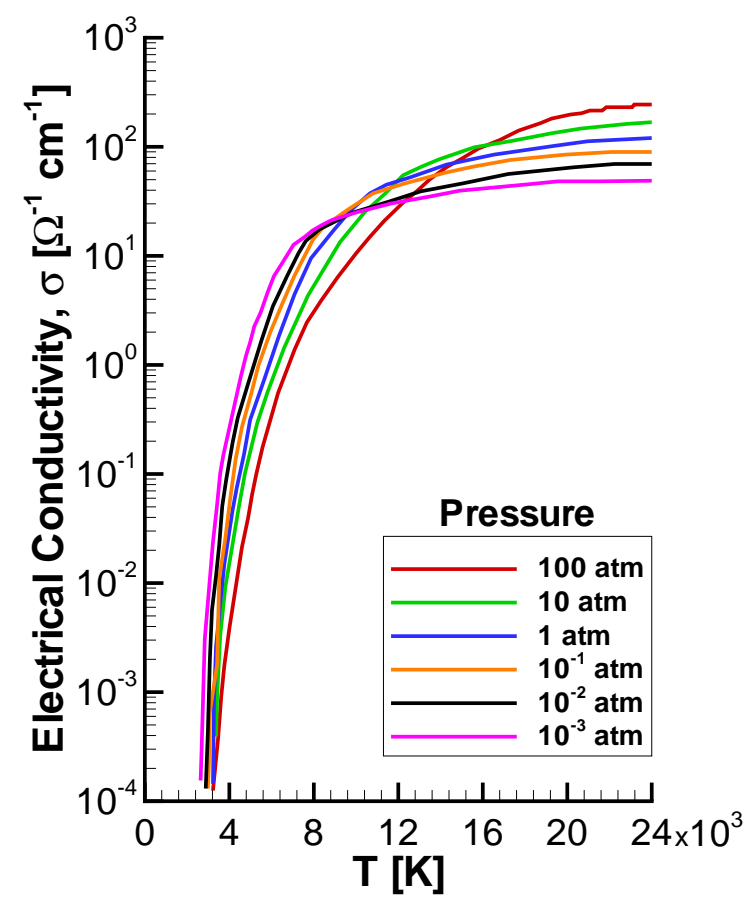

Figure 4. Electrical conductivity of equilibrium air for various temperatures and pressures reproduced from Ref. 31.

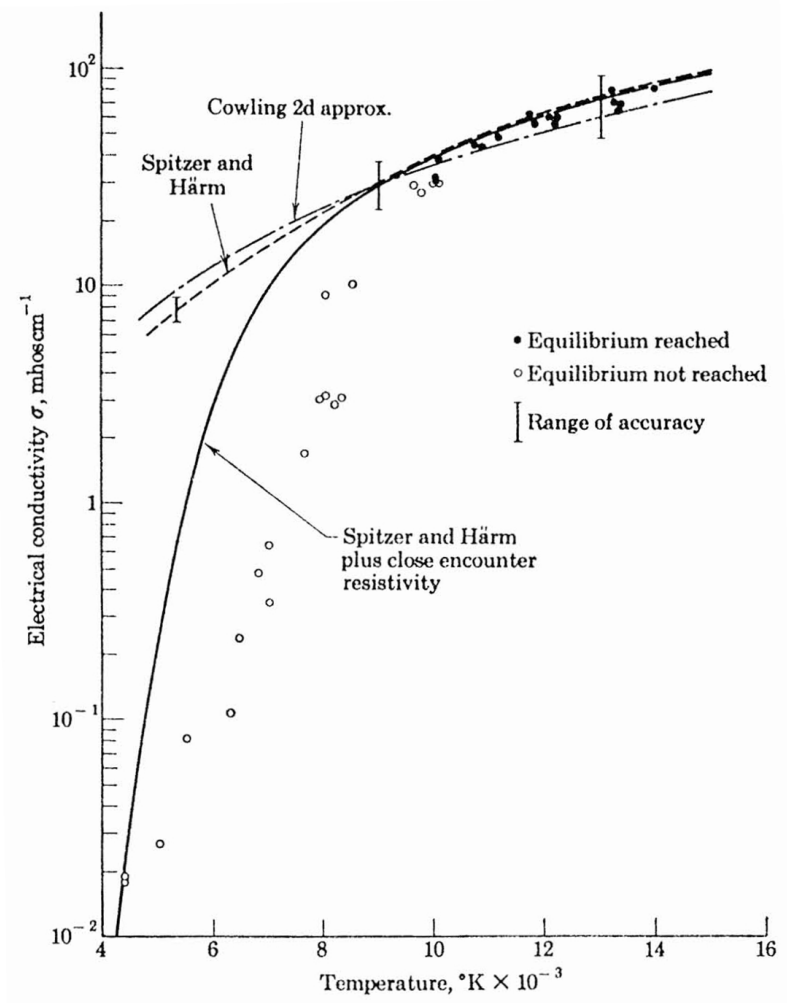

Figure 5. Electrical conductivity of equilibrium argon at $\mathrm{p}=0.013$ atm from Ref. 41 . 


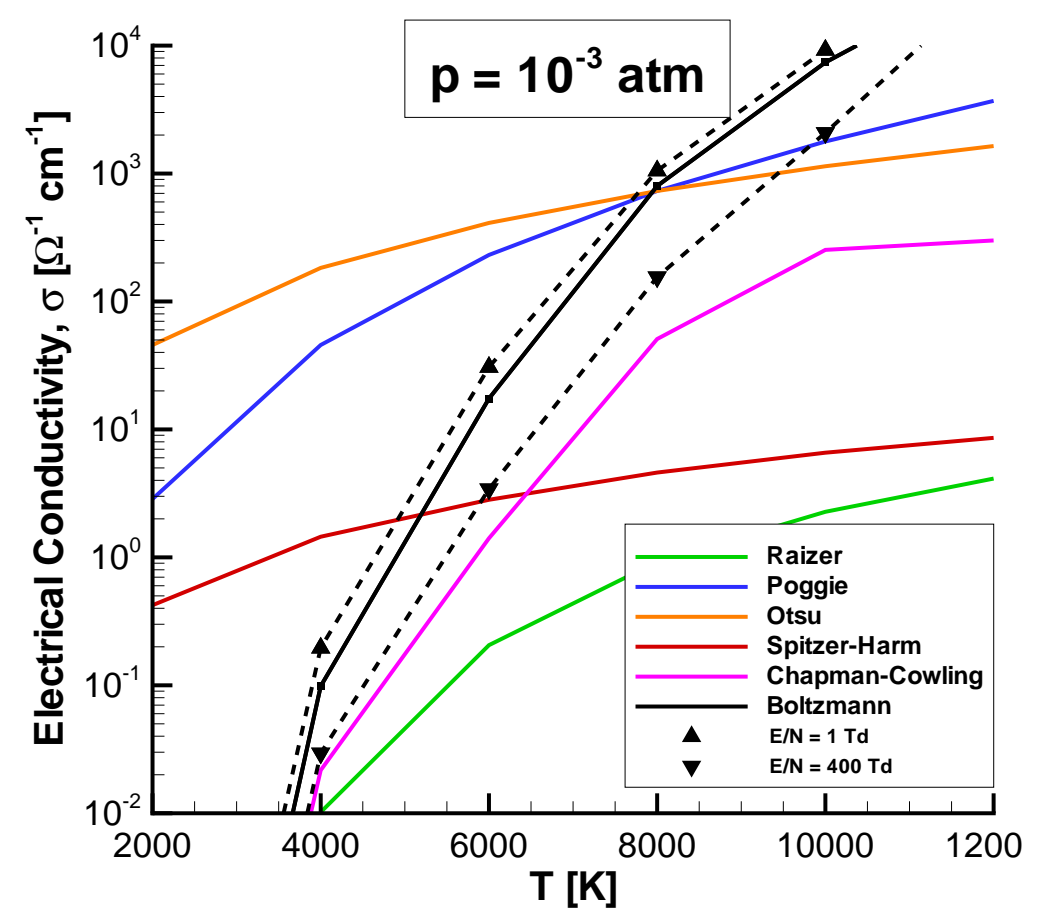

Figure 6. Electrical conductivity distribution versus temperature for various electrical conductivity models. $(p=0.001$ atm $)$

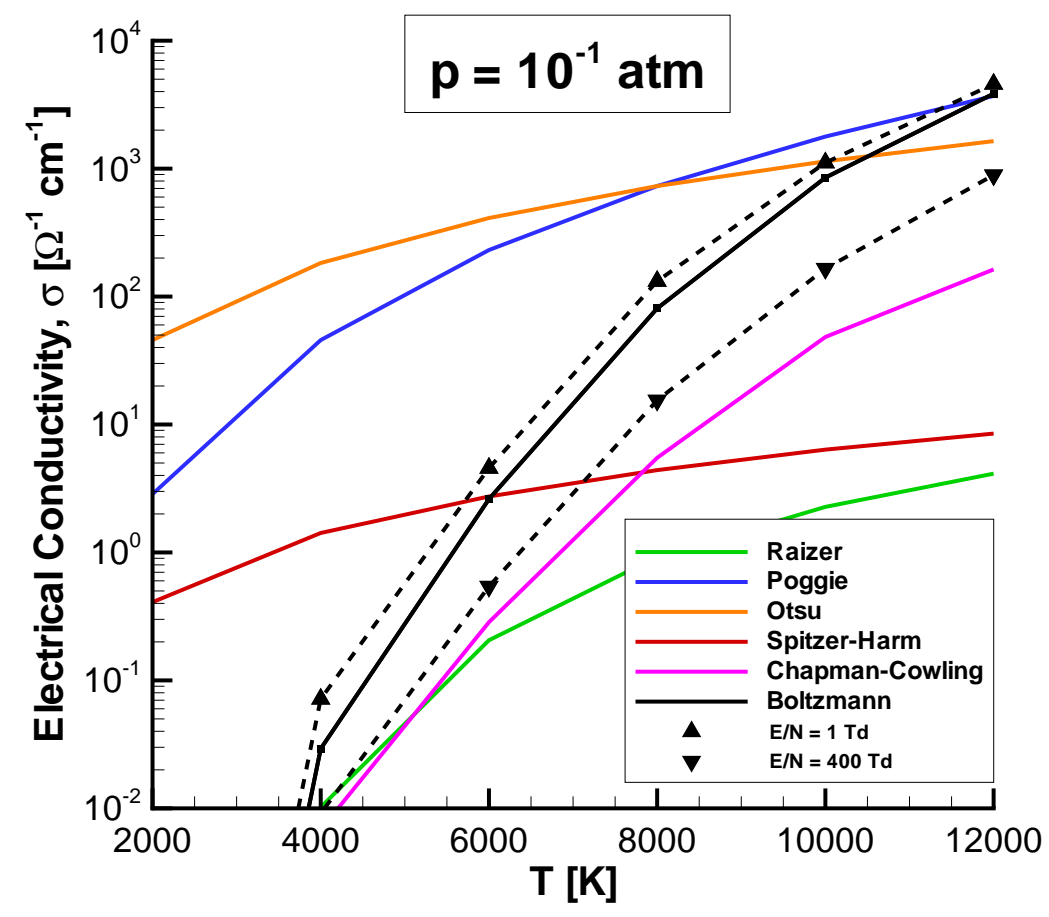

Figure 7. Electrical conductivity distribution versus temperature for various electrical conductivity models. $(\mathrm{p}=0.1 \mathrm{~atm})$ 


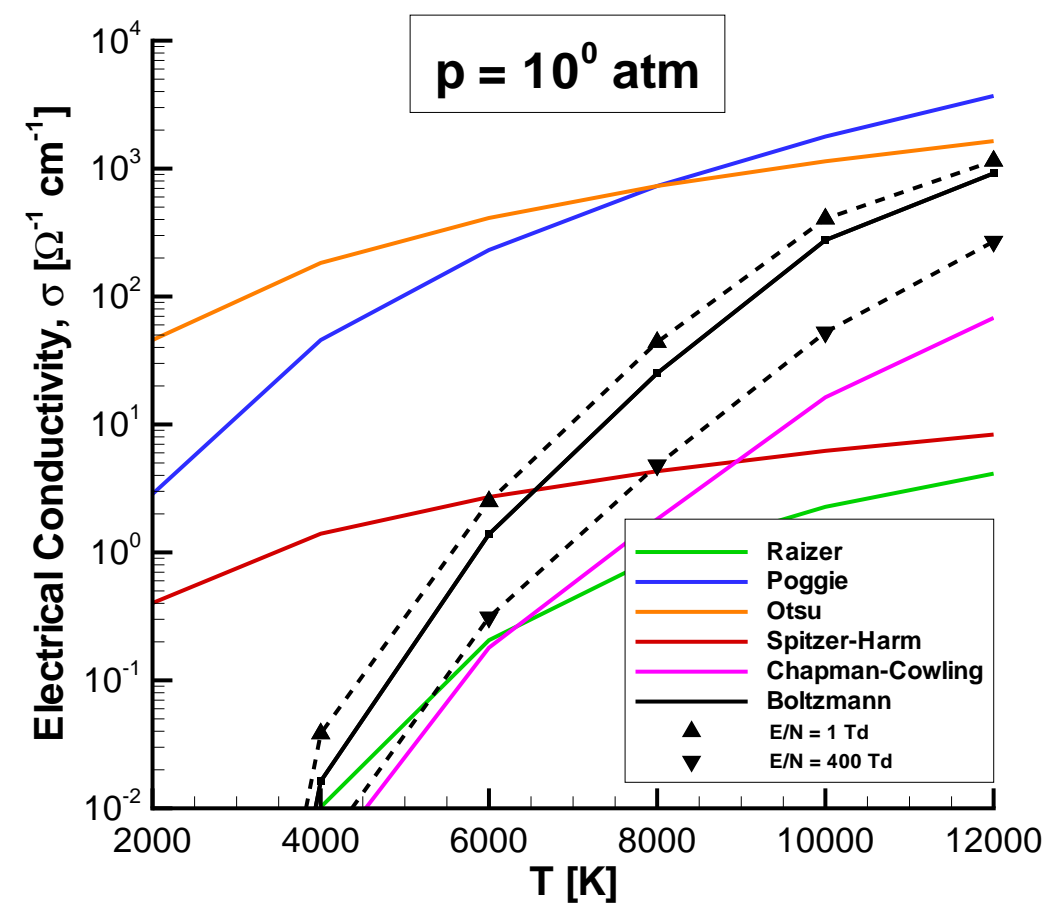

Figure 8. Electrical conductivity distribution versus temperature for various electrical conductivity models. $(p=1$ atm $)$ 


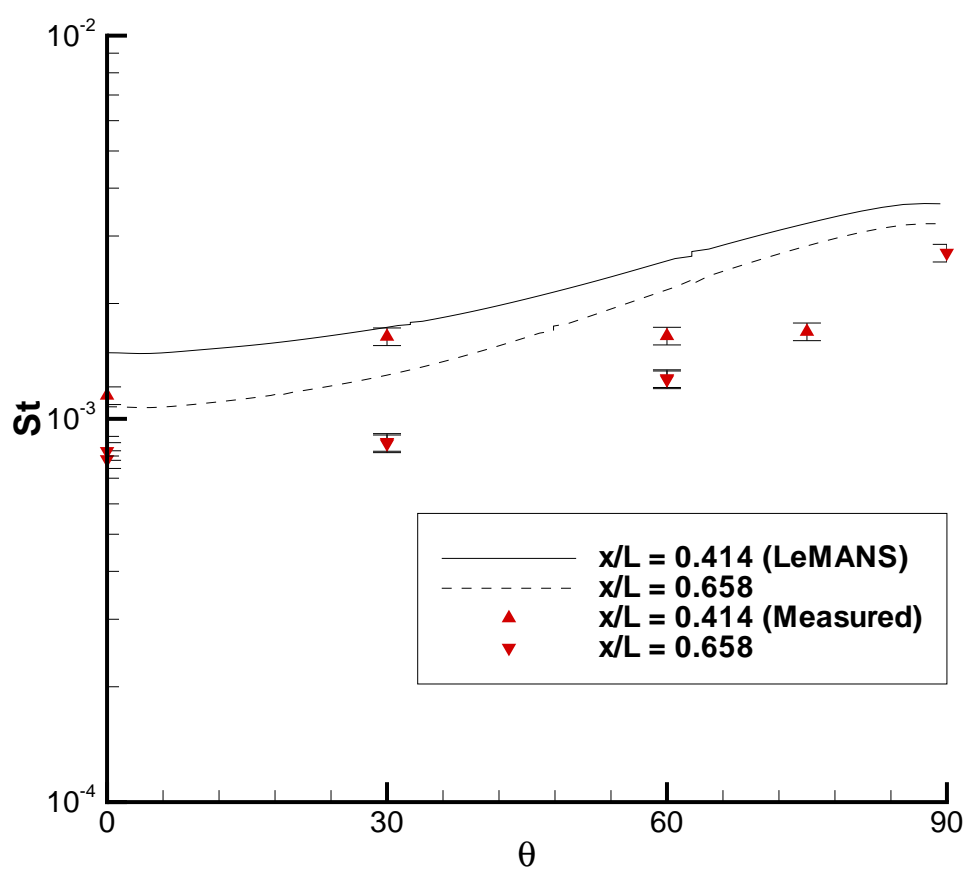

(a) Stanton number around the circumference

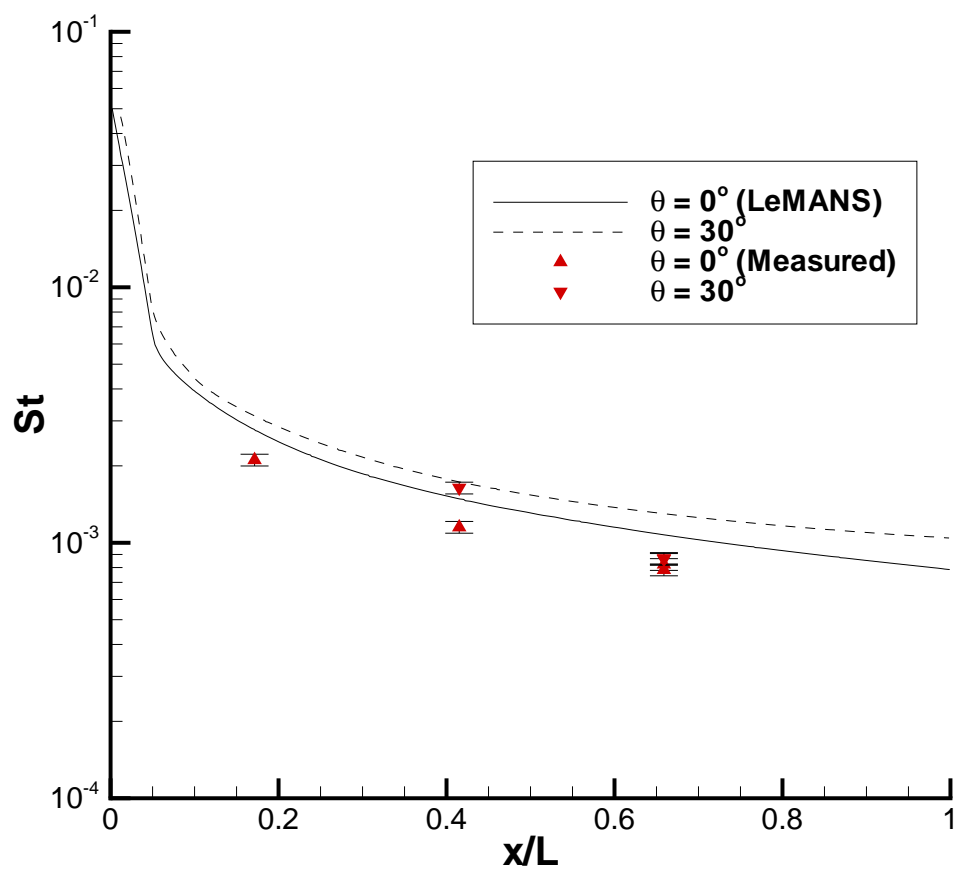

(b) Stanton number along rays

Figure 9. Stanton number distributions for Mach 14 blunt elliptic cone $( \pm 4.5$ percent experimental uncertainty). ${ }^{51}$ 


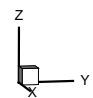

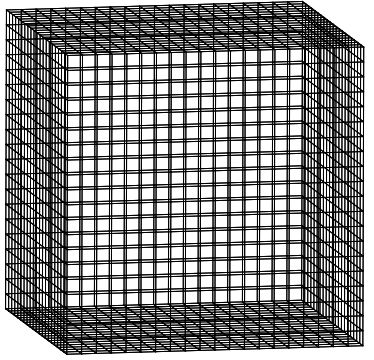

(a) Uniform

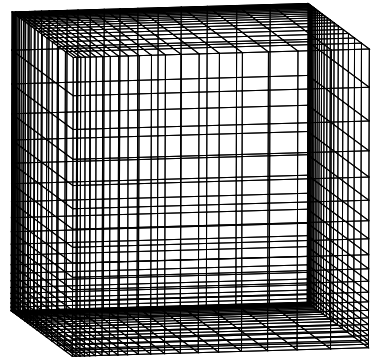

(b) Nonuniform

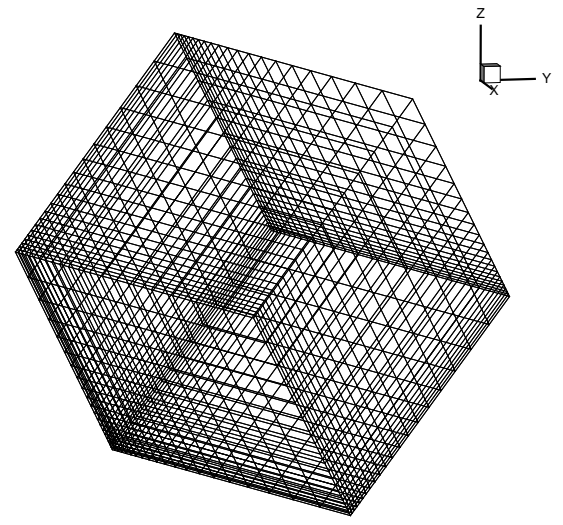

(c) Nonuniform (rotated $30^{\circ}$ along each axis)

Figure 10. Sample rectilinear meshes used in the analytical MHD validation case. $(20 \times 20 \times 20)$

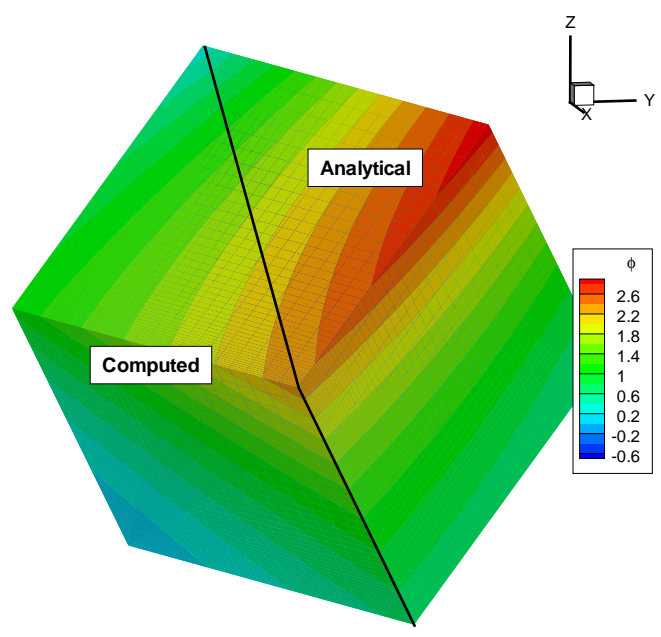

Figure 11. Contours of the electric potential $\phi$ for the analytical MHD validation case. The left side of the domain is composed of computed values while the right side is theoretical. $(20 \times 20 \times 20)$ 


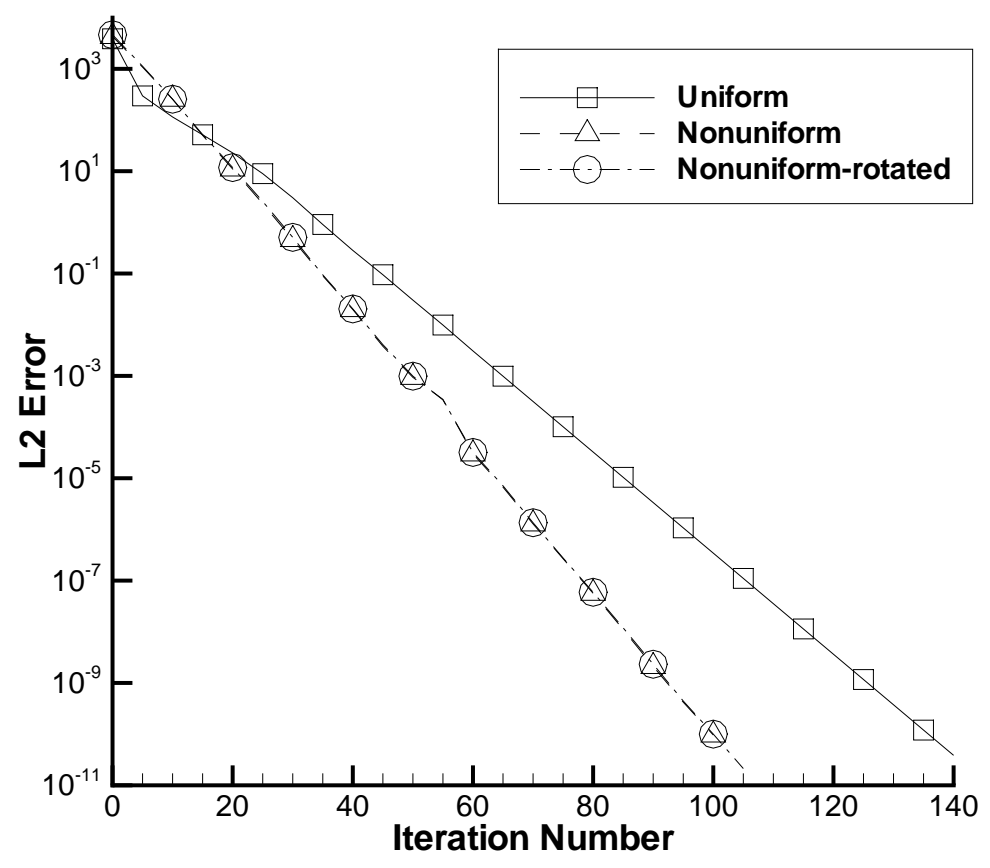

Figure 12. Least Square Normal versus iteration number for the various meshes used in the analytical validation case. Computations where made using the SOR method with $\omega=1.70$. $(20 \times 20 \times 20)$

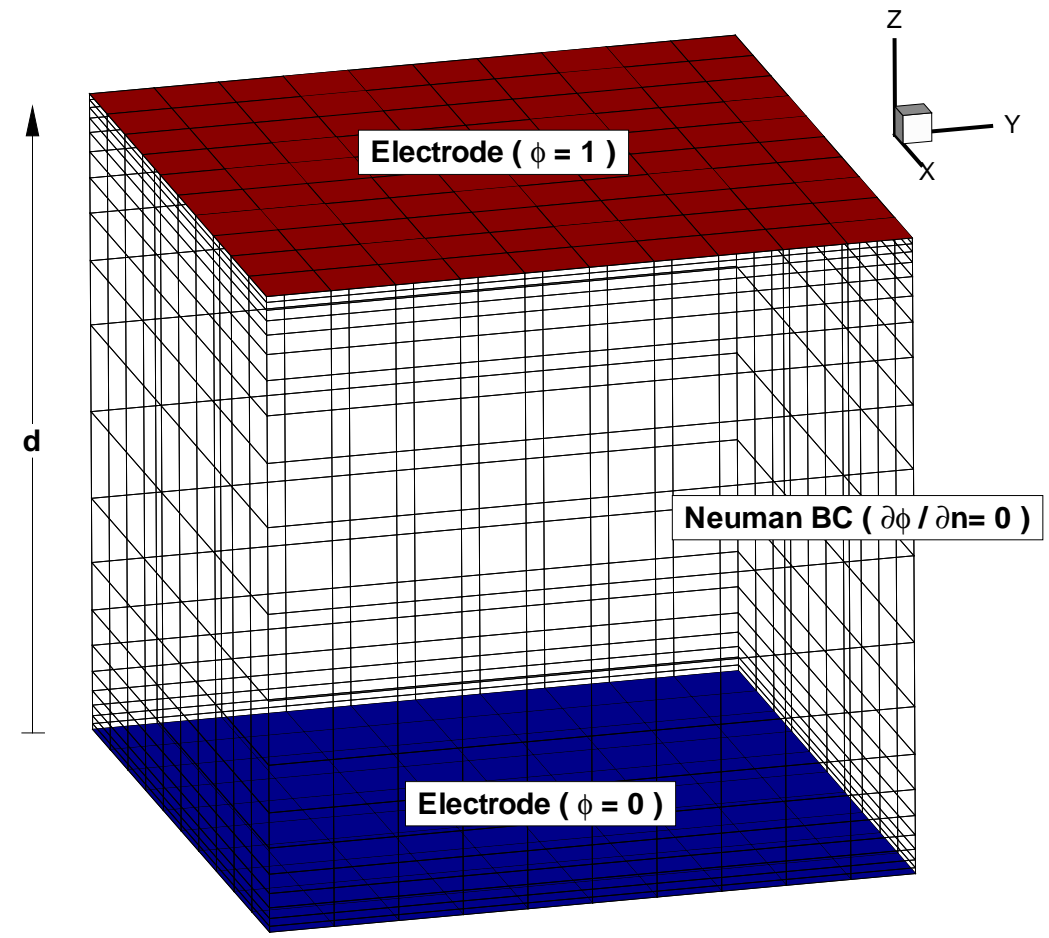

Figure 13. Nonuniform mesh for current flow between parallel electrode plates. $(10 \times 10 \times 20)$ 


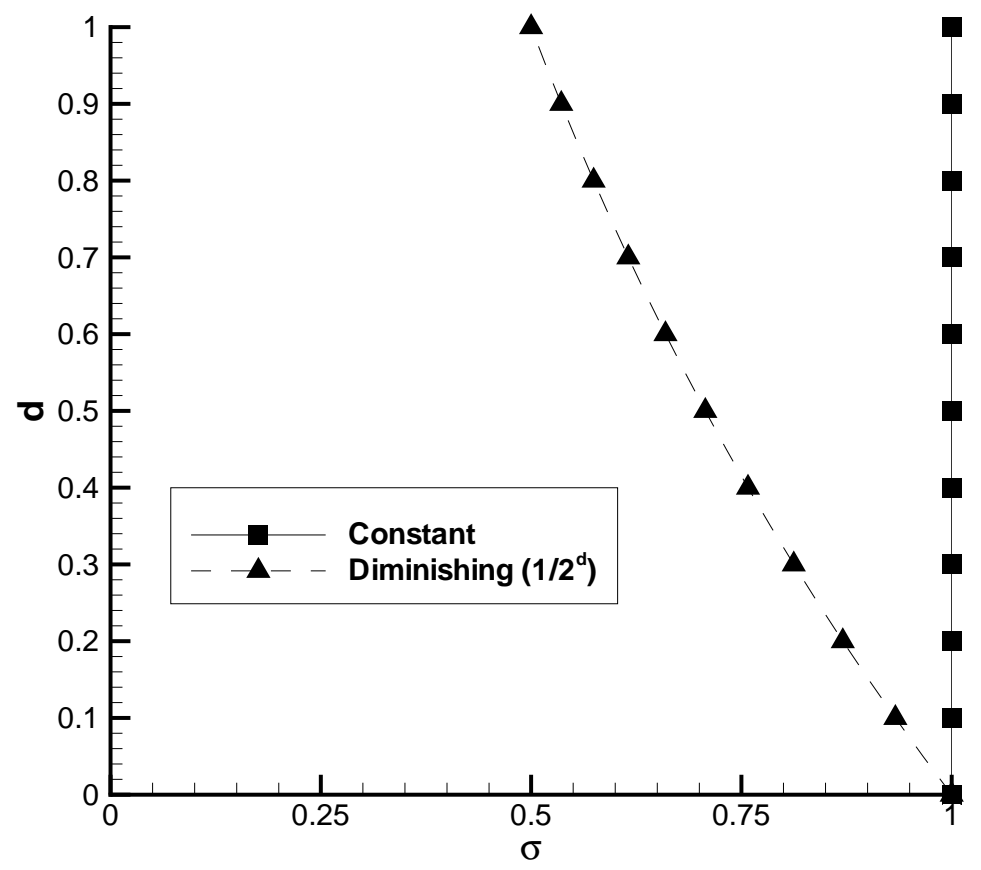

Figure 14. Various conductivity models used in flow between parallel electrode plates.

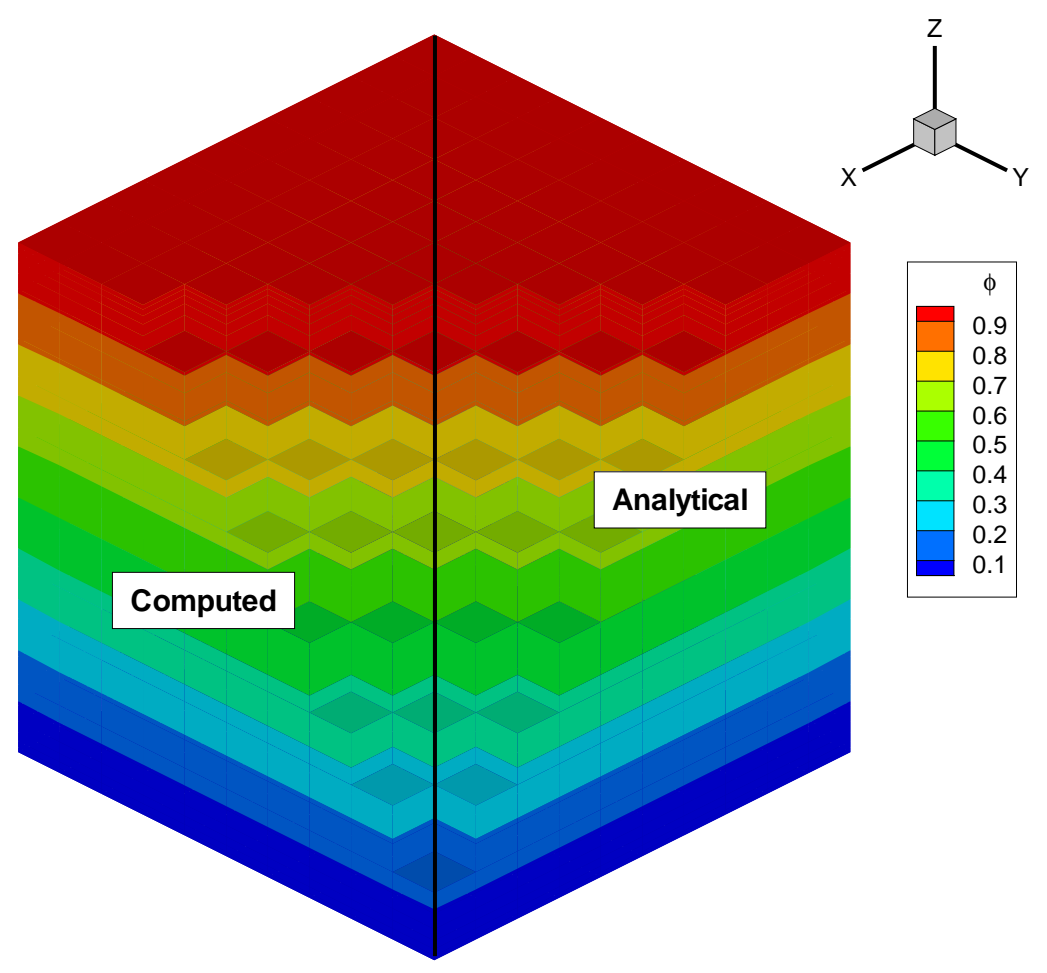

Figure 15. Contours of the electric potential $\phi$ for parallel electrodes with no flow $(\sigma=$ Constant $)$. The left side of the domain is composed of computed values while the right side is theoretical. $(10 \times 10 \times 20)$ 


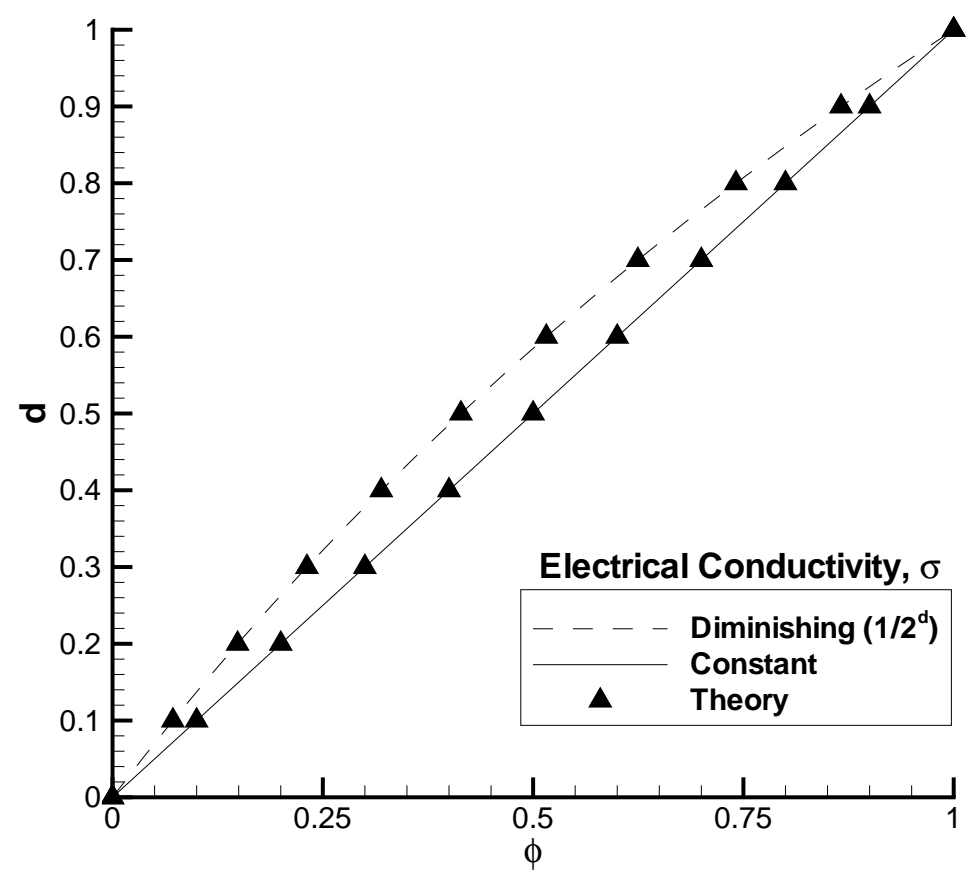

Figure 16. Electric potential distributions between parallel electrode plates with no flow. Two electrical conductivity models are shown $\left(\sigma=1\right.$ and $\left.\sigma=1 / 2^{d}\right)$. The distribution is extracted along $x=0.5, y=0.5$. 